\title{
Plasma Accelerators for Nuclear Applications and Materials Analyses (PANAMA)
}

\author{
P. Bots ${ }^{1}$, D.A. JAROSZYNSKI ${ }^{2}$, S.M. WIGGINS ${ }^{2}$, M. \\ SHAHZAD $^{2}$, J.C. RENSHAW ${ }^{1}$ \\ ${ }^{1}$ Department of Civil and Environmental Engineering, \\ University of Strathclyde, Glasgow, G1 1XJ, UK \\ ${ }^{2}$ Department of Physics, University of Strathclyde, Glasgow, \\ G4 0NG, UK
}

Understanding the (geo)chemical behaviour of nuclear materials and radionuclides is critical for safe operation at all stages of the nuclear fuel cycle, particularly in light of a renewed interest in nuclear energy to achieve the $\mathrm{CO}_{2}$ emissions targets in the Paris agreement. ${ }^{1}$ However, many nuclear materials are extremely dense and/or highly radioactive. Additionally, (geo)chemical reactions can occur on ultra-short time scales. These properties can severely limit analysis of these materials and processes.

Laser-driven plasma accelerators show great potential for the development of advanced analytical technologies that overcome such limitations. In such accelerators femtosecond (fs) laser pulses interact with a gas target to produce a plasma. The force of the laser pulse creates a plasma density wave in its wake, which accelerates electrons up to $\mathrm{GeV}$ energies in millimetres. These accelerated electrons can produce fs $\gamma$ - and $\mathrm{X}$-ray pulses (up to several MeV). ${ }^{2}$ Similarly, laser interaction with solid targets can produce proton and ion pulses. ${ }^{3}$

In this presentation we will present the progress and case studies on research possibilities for the nuclear energy sector at the Scottish Centre for Applications in Plasma Accelerators (SCAPA). ${ }^{4}$ At SCAPA we are developing capabilities exploiting radiation and particle pulses from laser-driven plasma accelerators. (1) For microstructural imaging, high energy radiation pulses can be used for $\gamma$ - and X-ray tomography of dense and large objects, including radioactive samples. (2) At the molecular scale, ultrafast radiation pulses can be utilised for time resolved X-ray diffraction and absorption spectroscopy. (3) The ultrafast $\gamma$ - and X-ray pulses can be synchronized with proton and ion beams for pumpprobe studies on the (fs) mechanisms of particle induced material damage. ${ }^{2,4}$ Such (combination of) capabilities will address fundamental research needs across nuclear sectors.

\section{References}

[1] Meeting the Energy Challenge, A White Paper on Nuclear Power. (2008). [2] Albert \& Thomas Plasma Physics and Controlled Fusion 58103001 (2016). [3] Higginson et al. Nature Communications 9724 (2018). [4] www.scapa.ac.uk [5] Mahieu et al. Nature Communications 93276 (2018). 